\title{
On the Markov-Modulated Insurance Risk Model with Tax
}

\author{
Jiaqin $\mathrm{Wei}^{a}$, Hailiang Yang ${ }^{b, *}$, Rongming Wang ${ }^{a, c}$ \\ ${ }^{a}$ School of Finance and Statistics, East China Normal University, Shanghai, 200241, China \\ ${ }^{b}$ Department of Statistics and Actuarial Science, The University of Hong Kong, Pokfulam Road, \\ Hong Kong, China \\ ${ }^{c}$ School of Mathematics and System Sciences, Shandong University, Jinan, 250100, China
}

\begin{abstract}
In this paper, we consider the Markov-modulated insurance risk model with tax. We assume that the claim inter-arrivers, claim sizes and premium process are influenced by an external Markovian environment process. The considered tax rule, which is the same with the one considered by Albrecher and Hipp [Albrecher, H., Hipp, C., 2007. Lundberg's risk process with tax. Blätter der DGVFM 28(1), 13-28], is to pay a certain proportion of the premium income, whenever the insurer is in a profitable situation. A system of differential equations of the non-ruin probabilities, given the initial environment state, are established in terms of the ruin probabilities under the Markov-modulated insurance risk model without tax. Furthermore, given the initial state, the differential equations satisfied by the expected accumulated discounted tax until ruin are also derived. We also give the analytical expressions for them by iteration methods.
\end{abstract}

Key words. Markov-modulated risk model; Tax payment; Ruin probability; Integro-differential equation

Email addresses: jiaqinwei@gmail.com (J.Q. Wei), hlyang@hkusua.hku.hk (H.L. Yang), rmwang@stat.ecnu.edu.cn (R.M. Wang).

*Corresponding author. 


\section{Introduction}

Ruin theory has been one of the main research topics in actuarial science since the publications of Lundberg (1909) and Cramér (1930). There is a huge amount of literature on ruin theory. The classical Lundberg problem has been extended in various ways. In recent years, one interesting development is to consider more general ruin functions rather than the ruin probability. In a seminal paper (see Gerber and Shiu (1998)), Gerber and Shiu introduced the expected discounted penalty function to provide a unified treatment of the time of ruin, the surplus before ruin and the deficit at ruin. The expected discounted penalty function is also called Gerber-Shiu function in the literature.

Another extension of the classical Lundberg problem is to consider more general and reasonable models. Recently, the Markov-modulated insurance risk model becomes popular. This model was proposed by Asmussen (1989) in which the claim inter-arrivers and claim sizes are influenced by an external environment process $\{J(t)\}_{t \geq 0}$. This model can capture the feature that insurance policies may need to change if economical or political environment changes (see Zhu and Yang (2007)). There has been considerable interest in this model. Lu and Li (2005) study ruin probabilities under this model. Ng and Yang (2006) presents some explicit results for the joint distribution of surplus before ruin and at ruin. Li and Lu (2007), Zhu and Yang (2007) and Lu and Li (2008) consider the Markovmodulated risk model with a dividend strategy. $\mathrm{Li}$ and $\mathrm{Lu}$ (2008) study the expected discounted penalty functions (Gerber-Shiu function) and their decompositions and the dividends-penalty identity under this model.

Suppose that $\{J(t)\}_{t \geq 0}$ is a homogenous continuous-time Markov chain taking values in a finite set $\mathbb{J}=\{1,2, \cdots, d\}$ with generator $\mathbf{Q}=\left(q_{i j}\right)_{d \times d}$. We further assume that $\{J(t)\}_{t \geq 0}$ is irreducible and recurrent with the stationary distribution $\boldsymbol{\pi}=\left(\pi_{1}, \pi_{2}, \cdots, \pi_{d}\right)$.

At time $t$, given $J(t)=i$, the premium rate is $c_{i}$, claims arrive according to Poisson process with rate $\lambda_{i}$, and the size of the claim which arrives at time $t$ follows the distribution $F_{i}$ with density $f_{i}$ and mean $\mu_{i}$. We denote by $X_{n}$ and $S_{n}$, respectively, the size the arrival time of the $n$th claim. Given $\left\{J\left(S_{n}\right)\right\}_{n \in \mathbb{N}}$, the sequence of claim sizes $\left\{X_{1}, X_{2}, \cdots\right\}$ are assumed to be mutually independent and independent of $\left\{S_{n}\right\}_{n \in \mathbb{N}}$ and $\{J(t)\}_{t \geq 0}$. Define $N(t)=\max \left\{n \in \mathbb{N}: S_{n} \leq t\right\}$ as the number of claims up to time $t$. The counting process $\{N(t)\}_{t \geq 0}$ is called a 
Markov-modulated Poisson process, which is a special case of the Cox process.

Suppose the initial surplus is $u \geq 0$, the corresponding surplus process $\{R(t)\}_{t \geq 0}$ is given by

$$
R(t)=u+C(t)-\sum_{n=1}^{N(t)} X_{n}, \quad t \geq 0,
$$

where $C(t)$ denotes the aggregate premium received during interval $(0, t]$. Let $T_{n}$ be the time at which the $n$th transition of the environment process $\{J(t)\}_{t \geq 0}$ occurs and $J_{n}$ be the state of the environment after its $n$th transition. Reinhard (1984) shows that

$$
C(t)=\sum_{k=1}^{M(t)} c_{J_{k-1}}\left(T_{k}-T_{k-1}\right)+c_{J_{M(t)}}\left(t-T_{M(t)}\right), \quad t \geq 0,
$$

where $M(t)=\max \left\{n \in \mathbb{N}: T_{n} \leq t\right\}$. From Reinhard (1984) ( see also $\mathrm{Ng}$ and Yang (2006)), the condition of having a positive expected profit is

$$
\sum_{i=1}^{d} \pi_{i}\left(c_{i}-\lambda_{i} \mu_{i}\right)>0
$$

Let $\tau=\inf \{t>0: R(t)<0\}$ be the time of ruin $(\tau=\infty$, if ruin does not happen). Now define the ultimate ruin probabilities, given the initial environment state is $i$, i.e. $J(0)=i$, by

$$
\Psi_{i}(u)=\mathrm{P}\{\tau<\infty \mid R(0)=u, J(0)=i\}, \quad i \in \mathbb{J}, u \geq 0 .
$$

The corresponding ultimate survival probabilities is defined by $\Phi_{i}(u)=1-\Psi_{i}(u)$.

Reinhard (1984) derives a system of integro-differential equations for the nonruin probabilities, $\Phi_{i}(u)$, for $i=1,2, \cdots, d$ :

$$
c_{i} \Phi_{i}^{\prime}(u)=\lambda_{i} \Phi_{i}(u)-\lambda_{i} \int_{0}^{u} \Phi_{i}(u-x) d F_{i}(x)-\sum_{j=1}^{d} q_{i j} \Phi_{j}(u), \quad u \geq 0
$$

where $q_{i}=-q_{i i}$. The equation (1.3) has a unique solution such that $\Phi_{i}(\infty)=1$, for $i \in \mathbb{J}$. For more about the solution, see Lu and Li (2005).

Albrecher and Hipp (2007) investigated how tax influences the behavior of the ultimate ruin probability under the classical Lundberg's risk model. They assume that the tax is paid at a fixed rate $\gamma \in(0,1)$ of the insurer's income (premia) whenever he is in a profitable situation, defined as being at a running 
maximum of the surplus process. In this paper, we extend their results to the Markov-modulated risk model which has been specified previously.

We denote by $\gamma$ the vector of tax rates, i.e. $\gamma=\left(\gamma_{1}, \gamma_{2}, \cdots, \gamma_{d}\right)^{T}$, and by $R(t ; \gamma)$ the Markov-modulated risk process with tax rate $\gamma$. At time $t$, given $J(t)=i$, we assume that the tax is paid at rate $\gamma_{i} \in(0,1)$ of the insurer's income (i.e. the premium $c_{i}$ ), if the insurer is in the profitable situation at time $t$, i.e., $R(t ; \gamma)=\max \{R(u, \gamma): u \leq t\}$. Let $\tau(\gamma)=\inf \{t>0: R(t ; \gamma)<0\}$ be the time of ruin. Then the the ultimate ruin probabilities, given the initial environment state $i$, can be defined by

$$
\Psi_{i}(u ; \gamma)=\mathrm{P}\{\tau(\gamma)<\infty \mid R(0 ; \gamma)=u, J(0)=i\}, \quad i \in \mathbb{J}, u \geq 0
$$

Similarly, the corresponding survival probabilities is defined by $\Phi_{i}(u ; \gamma)=1-$ $\Psi_{i}(u ; \gamma)$

This paper is organized as follows. The non-ruin probabilities are studied in the next section. A system of differential equations satisfied by the non-ruin probabilities, given the initial environment state, are established in terms of the ruin probabilities under the Markov-modulated risk model without tax. In Section 3 , the expected accumulated discounted tax until ruin is considered, a system of differential equation is derived. Finally, we give the analytical expressions by iteration methods.

\section{Ruin probability}

Let us begin this section by showing how the risk process $R(t ; \gamma)$ evolves. If $R(0 ; \gamma)=u$, then there is a period with profit in which tax must be paid until the first claim arrives at time $W_{1}$ and has size $Y_{1}$. Obviously, $W_{1}=S_{1}$ and $Y_{1}=X_{1}$. Then the gains level is set to

$L_{1}=u+\sum_{k=1}^{M\left(W_{1}\right)} c_{J_{k-1}}\left(1-\gamma_{J_{k-1}}\right)\left(T_{k}-T_{k-1}\right)+c_{J_{M\left(W_{1}\right)}}\left(1-\gamma_{J_{M\left(W_{1}\right)}}\right)\left(W_{1}-T_{M\left(W_{1}\right)}\right)$

Then there is a period in which the insurer does not pay the tax until the risk process reaches $L_{1}$ again, say at time $\sigma_{1}$. We have a period with profit until the first claim after time $\sigma_{1}$, which happens at $\sigma_{1}+W_{2}$ and has size $Y_{2}$. Note that there is some $n \in \mathbb{N}$ such that $\sigma_{1}+W_{2}=S_{n}$ and $Y_{2}=X_{n}$. The new gains level is 
set to

$$
\begin{aligned}
L_{2}= & L_{1}+c_{J_{M\left(\sigma_{1}\right)}}\left(1-\gamma_{J_{M\left(\sigma_{1}\right)}}\right)\left(T_{M\left(\sigma_{1}\right)+1}-\sigma_{1}\right) \\
& +\sum_{k=M\left(\sigma_{1}\right)+2}^{M\left(\sigma_{1}+W_{2}\right)} c_{J_{k-1}}\left(1-\gamma_{J_{k-1}}\right)\left(T_{k}-T_{k-1}\right) \\
& +c_{J_{M\left(\sigma_{1}+W_{2}\right)}}\left(1-\gamma_{J_{M\left(\sigma_{1}+W_{2}\right)}}\right)\left(\sigma_{1}+W_{2}-T_{M\left(\sigma_{1}+W_{2}\right)}\right),
\end{aligned}
$$

and so on. Let $\sigma_{0}=0$ and $L_{0}=u$, then for $n \geq 1$, we have

$$
\begin{aligned}
\sigma_{n}= & \inf \left\{t>\sigma_{n-1}+W_{n}: R(t ; \gamma)=L_{n}\right\} \\
W_{n}= & \inf \left\{t>0: N\left(\sigma_{n-1}+t\right)>N\left(\sigma_{n-1}\right)\right\} \\
Y_{n}= & R\left(\sigma_{n-1}+W_{n}-; \gamma\right)-R\left(\sigma_{n-1}+W_{n} ; \gamma\right) \\
L_{n}= & L_{n-1}+c_{J_{M\left(\sigma_{n-1}\right)}}\left(1-\gamma_{\left.J_{M\left(\sigma_{n-1}\right)}\right)}\right)\left(T_{M\left(\sigma_{n-1}\right)+1}-\sigma_{n-1}\right) \\
& \quad+\sum_{k=M\left(\sigma_{n-1}\right)+2}^{M\left(\sigma_{n-1}+W_{n}\right)} c_{J_{k-1}}\left(1-\gamma_{J_{k-1}}\right)\left(T_{k}-T_{k-1}\right) \\
& +c_{J_{M\left(\sigma_{n-1}+W_{n}\right)}}\left(1-\gamma_{J_{M\left(\sigma_{n-1}+W_{n}\right)}}\right)\left(\sigma_{n-1}+W_{n}-T_{M\left(\sigma_{n-1}+W_{n}\right)}\right) .
\end{aligned}
$$

The time intervals with profit are $\left(\sigma_{n-1}, \sigma_{n-1}+W_{n}\right), n \geq 1$. The intervals without profit are

$$
\mathscr{I}_{n}=\left[\sigma_{n-1}+W_{n}, \sigma_{n}\right), \quad n \geq 1
$$

It is easy to see that ruin happens for the process $R(t ; \gamma)$ only if $R(t ; \gamma)<0$ for some $t \in \mathscr{I}_{n}, n \geq 1$. With the notation $U(t)=\sup \left\{n \in \mathbb{N}: \sigma_{n-1}+W_{n} \leq t\right\}$, we can rewrite the surplus as

$$
R(t ; \gamma)= \begin{cases}R_{1}(t ; \gamma), & \sigma_{U(t)-1}+W_{U(t)} \leq t \leq \sigma_{U(t)} \\ R_{2}(t ; \gamma), & \sigma_{U(t)}<t<\sigma_{U(t)}+W_{U(t)+1}\end{cases}
$$

where

$$
\begin{aligned}
R_{1}(t ; \gamma)= & L_{U(t)}+c_{J_{M\left(\sigma_{U(t)-1}+W_{U(t)}\right)}}\left(T_{M\left(\sigma_{U(t)-1}+W_{U(t)}\right)+1}-\sigma_{U(t)-1}-W_{U(t)}\right) \\
& +\sum_{k=M\left(\sigma_{U(t)-1}+W_{U(t)}\right)+2}^{M(t)} c_{J_{k-1}}\left(T_{k}-T_{k-1}\right) \\
& +c_{J_{M(t)}}\left(t-T_{M(t)}\right)-\sum_{i=N\left(\sigma_{U(t)-1}+W_{U(t)}\right)}^{N(t)} Y_{i},
\end{aligned}
$$


and

$$
\begin{aligned}
R_{2}(t ; \gamma)= & L_{U(t)}+c_{\left.J_{M\left(\sigma_{U}(t)\right.}\right)}\left(1-\gamma_{\left.J_{M\left(\sigma_{U}(t)\right.}\right)}\right)\left(T_{M\left(\sigma_{U(t)}\right)+1}-\sigma_{U(t)}\right) \\
& +\sum_{k=M\left(\sigma_{U(t)}\right)+2}^{M(t)} c_{J_{k-1}}\left(1-\gamma_{J_{k-1}}\right)\left(T_{k}-T_{k-1}\right) \\
& +c_{J_{M(t)}}\left(1-\gamma_{J_{M(t)}}\right)\left(t-T_{M(t)}\right) .
\end{aligned}
$$

Lemma 2.1. For $u \geq 0$ and $i \in \mathbb{J}$, if the condition

$$
\sum_{i=1}^{d} \pi_{i}\left(c_{i}\left(1-\gamma_{i}\right)-\lambda_{i} \mu_{i}\right)>0
$$

holds, then $\Psi_{i}(u ; \gamma)<1$.

Proof. Define a new risk process

$$
\begin{aligned}
\tilde{R}(t ; \gamma)= & L_{U(t)} \\
& +c_{J_{M\left(\sigma_{U(t)-1}+W_{U(t)}\right)}}\left(1-\gamma_{\left.J_{M\left(\sigma_{U(t)-1}+W_{U(t)}\right)}\right)}\right)\left(T_{M\left(\sigma_{U(t)-1}+W_{U(t)}\right)+1}-\sigma_{U(t)-1}-W_{U(t)}\right) \\
& +\sum_{k=M\left(\sigma_{U(t)-1}+W_{U(t)}\right)+2} c_{J_{k-1}}\left(1-\gamma_{J_{k-1}}\right)\left(T_{k}-T_{k-1}\right)+c_{J_{M(t)}}\left(1-\gamma_{J_{M(t)}}\right)\left(t-T_{M(t)}\right) \\
& -\sum_{i=1}^{N(t)} Y_{i},
\end{aligned}
$$

and let $\tilde{\tau}(\gamma)$ be the corresponding time to ruin. From (2.1), it is easy to see

$$
\tilde{R}(t ; \gamma)=u+\tilde{C}(t ; \gamma)-\sum_{i=1}^{N(t)} Y_{i}
$$

where

$$
\tilde{C}(t ; \gamma)=\sum_{k=1}^{M(t)} c_{J_{k-1}}\left(1-\gamma_{J_{k-1}}\right)\left(T_{k}-T_{k-1}\right)+c_{J_{M(t)}}\left(1-\gamma_{J_{M(t)}}\right)\left(t-T_{M(t)}\right) .
$$

Since $\tilde{R}(t ; \gamma) \leq R_{1}(t ; \gamma) \leq R_{2}(t ; \gamma)$, we have

$$
\mathrm{P}\{\tau(\gamma)<\infty\} \leq \mathrm{P}\{\tilde{\tau}(\gamma)<\infty\}<1
$$

Proof of the last inequality can be found in Reinhard (1984) (see also (1.2)). 
For $0 \leq u \leq l$, let $\tau_{l}$ be the first time that the surplus $R(t)$ reaches level $l$, and define

$$
p_{i j}(u, l)=\mathrm{P}\left\{\tau_{l}<\tau, J\left(\tau_{l}\right)=j \mid R(0)=u, J(0)=i\right\}, \quad i, j \in \mathbb{J},
$$

to be the probability that the surplus process $R(t)$ attains level $l$ at state $j$ from initial state $i$ and initial surplus $u$ without ruin.

Clearly, $p_{i j}(l, l)=\chi(i=j)$ for $i, j \in \mathbb{J}$, where $\chi(\cdot)$ is the indicator function. And from $\mathrm{Li}$ and $\mathrm{Lu}$ (2007), we have

$$
\mathbf{P}(u, l):=\left(p_{i j}(u, l)\right)_{d \times d}=\mathbf{v}(u)[\mathbf{v}(l)]^{-1}
$$

where $\mathbf{v}(u)=\left(v_{i j}(u)\right)_{d \times d}$ is an matrix whose columns are particular solutions to the following system of integro-differential equations:

$$
c_{i} v_{i}^{\prime}(u)=\lambda_{i} v_{i}(u)-\lambda_{i} \int_{0}^{u} v_{i}(u-x) d F_{i}(x)-\sum_{j=1}^{d} q_{i j} v_{j}(u),
$$

with boundary conditions $\mathbf{v}(0)=\mathbf{I}$.

Theorem 2.1. For each fixed $i \in \mathbb{J}$ and $u \geq 0, \Phi_{i}(u ; \gamma)$ satisfies the following integro-differential equation:

$$
\begin{aligned}
c_{i}\left(1-\gamma_{i}\right) \Phi_{i}^{\prime}(u ; \gamma)= & \lambda_{i} \Phi_{i}(u ; \gamma)-\lambda_{i} \int_{0}^{u} \sum_{j=1}^{d} p_{i j}(u-x, u) \Phi_{j}(u ; \gamma) d F_{i}(x) \\
& -\sum_{j=1}^{d} q_{i j} \Phi_{j}(u ; \gamma) .
\end{aligned}
$$

Proof. Distinguish the following cases: (i) the first transition of the environment state occurs at time $t$ and the first claim arrives at time $s$ before time $t$; (ii) the first transition of the environment state occurs at time $t$ before the arrival of the first claim. By conditioning accordingly,

$$
\begin{aligned}
& \Phi_{i}(u ; \gamma)= \int_{0}^{\infty} q_{i} e^{-q_{i} t} \int_{0}^{t} \lambda_{i} e^{-\lambda_{i} s} \int_{0}^{u+c_{i}\left(1-\gamma_{i}\right) s} \\
& \sum_{j=1}^{d} p_{i j}\left(u+c_{i}\left(1-\gamma_{i}\right) s-x, u+c_{i}\left(1-\gamma_{i}\right) s\right) \Phi_{j}\left(u+c_{i}\left(1-\gamma_{i}\right) s ; \gamma\right) d F_{i}(x) d s d t \\
&+\int_{0}^{\infty} \lambda_{i} e^{-\lambda_{i} s} \int_{0}^{t} q_{i} e^{-q_{i} t} \sum_{j \neq i} \frac{q_{i j}}{q_{i}} \Phi_{j}\left(u+c_{i}\left(1-\gamma_{i}\right) t ; \gamma\right) d t d s \\
&= \int_{0}^{\infty} \lambda_{i} e^{-\left(\lambda_{i}+q_{i}\right) s} \int_{0}^{u+c_{i}\left(1-\gamma_{i}\right) s} \sum_{j=1}^{d} p_{i j}\left(u+c_{i}\left(1-\gamma_{i}\right) s-x, u+c_{i}\left(1-\gamma_{i}\right) s\right) \\
& \Phi_{j}\left(u+c_{i}\left(1-\gamma_{i}\right) s ; \gamma\right) d F_{i}(x) d s+\int_{0}^{\infty} e^{-\left(\lambda_{i}+q_{i}\right) t} \sum_{j \neq i} q_{i j} \Phi_{j}\left(u+c_{i}\left(1-\gamma_{i}\right) t ; \gamma\right) d t .
\end{aligned}
$$


Changing variables $w=u+c_{i}\left(1-\gamma_{i}\right) t$ (or $\left.w=u+c_{i}\left(1-\gamma_{i}\right) s\right)$ gives

$$
\begin{aligned}
\Phi_{i}(u ; \gamma)= & \int_{u}^{\infty} \frac{\lambda_{i}}{c_{i}\left(1-\gamma_{i}\right)} e^{-\left(\lambda_{i}+q_{i}\right) \frac{w-u}{c_{i}\left(1-\gamma_{i}\right)}} \int_{0}^{w} \sum_{j=1}^{d} p_{i j}(w-x, w) \Phi_{j}(w ; \gamma) d F_{i}(x) d w \\
& +\int_{u}^{\infty} \frac{1}{c_{i}\left(1-\gamma_{i}\right)} e^{-\left(\lambda_{i}+q_{i}\right) \frac{w-u}{c_{i}\left(1-\gamma_{i}\right)}} \sum_{j \neq i} q_{i j} \Phi_{j}(w ; \gamma) d w .
\end{aligned}
$$

Differentiating the above equation with respect to $u$ leads to (2.6).

With the notations

$$
\begin{aligned}
\mathbf{C}(\gamma) & :=\operatorname{diag}\left(c_{1}\left(1-\gamma_{1}\right), c_{2}\left(1-\gamma_{2}\right), \cdots, c_{d}\left(1-\gamma_{d}\right)\right), \\
\mathbf{\Lambda} & :=\operatorname{diag}\left(\lambda_{1}, \lambda_{2}, \cdots, \lambda_{d}\right), \\
\tilde{\boldsymbol{\Lambda}}(x) & :=\operatorname{diag}\left(\lambda_{1} f_{1}(x), \lambda_{2} f_{2}(x), \cdots, \lambda_{d} f_{d}(x)\right),
\end{aligned}
$$

(2.6) can be represented in matrix notation,

$$
\mathbf{C}(\gamma) \boldsymbol{\Phi}^{\prime}(u ; \gamma)=(\boldsymbol{\Lambda}-\mathbf{Q}) \boldsymbol{\Phi}(u ; \gamma)-\int_{0}^{u} \tilde{\boldsymbol{\Lambda}}(x) \mathbf{P}(u-x, u) d x \boldsymbol{\Phi}(u ; \gamma)
$$

where $\boldsymbol{\Phi}(u ; \gamma)=\left(\Phi_{1}(u ; \gamma), \Phi_{2}(u ; \gamma), \cdots, \Phi_{d}(u ; \gamma)\right)^{T}$.

Let

$$
\Psi_{i j}(u)=\mathrm{P}\{\tau<\infty, J(\tau)=j \mid R(0)=u, J(0)=i\}
$$

be the ruin probability of the surplus $R(t)$ if the ruin is caused by a claim in state $j$ given that the initial state is $i$ and we have for $i, j \in \mathbb{J}$,

$$
\Psi_{i}(u)=\sum_{j=1}^{d} \Psi_{i j}(u)
$$

From Li and Lu (2008), we have for $i \in \mathbb{J}$,

$$
c_{i} \Psi_{i i}^{\prime}(u)=\lambda_{i} \Psi_{i i}(u)-\lambda_{i}\left[\int_{0}^{u} \Psi_{i i}(u-x) d F_{i}(x)+\int_{u}^{\infty} d F_{i}(x)\right]-\sum_{k=1}^{d} q_{i k} \Psi_{k i}(u),
$$

and for $i \neq j$,

$$
c_{i} \Psi_{i j}^{\prime}(u)=\lambda_{i} \Psi_{i j}(u)-\lambda_{i} \int_{0}^{u} \Psi_{i j}(u-x) d F_{i}(x)-\sum_{k=1}^{d} q_{i k} \Psi_{k j}(u),
$$

or in matrix form

$$
\mathbf{C} \boldsymbol{\Psi}^{\prime}(u)=(\boldsymbol{\Lambda}-\mathbf{Q}) \boldsymbol{\Psi}(u)-\int_{0}^{u} \tilde{\boldsymbol{\Lambda}}(x) \boldsymbol{\Psi}(u-x) d x-\int_{u}^{\infty} \tilde{\boldsymbol{\Lambda}}(x) d x,
$$


where $\boldsymbol{\Psi}(u)=\left(\Psi_{i j}(u)\right)_{d \times d}$ and $\mathbf{C}=\operatorname{diag}\left(c_{1}, c_{2}, \cdots, c_{d}\right)$.

Next we are going to express (2.7) in terms of $\boldsymbol{\Psi}(u)$. Let $\tilde{\boldsymbol{\Psi}}(u)=\left(\tilde{\Psi}_{i j}(u)\right)_{d \times d}=$ $\mathbf{I}-\boldsymbol{\Psi}(u)$ with $\mathbf{I}$ being the $d \times d$ identity matrix. From Li and Lu (2008), we have for $i ; j \in \mathbb{J}$,

$$
c_{i} \tilde{\Psi}_{i j}^{\prime}(u)=\lambda_{i} \tilde{\Psi}_{i j}(u)-\lambda_{i} \int_{0}^{u} \tilde{\Psi}_{i j}(u-x) f_{i}(x) d x-\sum_{k=1}^{d} q_{i k} \tilde{\Psi}_{k j}(u)+q_{i j},
$$

or in matrix notation

$$
\mathbf{C} \tilde{\boldsymbol{\Psi}}^{\prime}(u)=(\boldsymbol{\Lambda}-\mathbf{Q}) \tilde{\boldsymbol{\Psi}}(u)-\int_{0}^{u} \tilde{\boldsymbol{\Lambda}}(x) \tilde{\mathbf{\Psi}}(u-x) d x+\mathbf{Q} .
$$

Further more, using the same method of Li and $\mathrm{Lu}$ (2008), we also have

$$
\mathbf{v}(u)=\tilde{\boldsymbol{\Psi}}(u)[\tilde{\boldsymbol{\Psi}}(0)]^{-1}-\int_{0}^{u} \tilde{\boldsymbol{\Psi}}(x)[\tilde{\boldsymbol{\Psi}}(0)]^{-1} \boldsymbol{\Delta} e^{-\boldsymbol{\Delta}(u-x)} d x
$$

where $\boldsymbol{\Delta}=\mathbf{C}^{-1} \mathbf{Q}[\tilde{\boldsymbol{\Psi}}(0)]^{-1}$.

With (2.4) and (2.10), (2.7) can be written as

$$
\begin{aligned}
\mathbf{C}(\gamma) \boldsymbol{\Phi}^{\prime}(u ; \gamma)= & (\boldsymbol{\Lambda}-\mathbf{Q}) \boldsymbol{\Phi}(u ; \gamma)-\left\{\int_{0}^{u} \tilde{\boldsymbol{\Lambda}}(x) \tilde{\boldsymbol{\Psi}}(u-x)[\tilde{\boldsymbol{\Psi}}(0)]^{-1} d x\right. \\
& \left.-\int_{0}^{u} \int_{0}^{u-t} \tilde{\boldsymbol{\Lambda}}(x) \tilde{\boldsymbol{\Psi}}(u-t-x)[\tilde{\boldsymbol{\Psi}}(0)]^{-1} d x \Delta e^{-\Delta t} d t\right\}[\mathbf{v}(u)]^{-1} \boldsymbol{\Phi}(u ; \gamma) .
\end{aligned}
$$

From (2.9) we have

$$
\mathbf{C}(\gamma) \boldsymbol{\Phi}^{\prime}(u ; \gamma)=(\boldsymbol{\Lambda}-\mathbf{Q}-\mathbf{A}(\mathbf{u})) \boldsymbol{\Phi}(u ; \gamma),
$$

where

$$
\begin{aligned}
\mathbf{A}(\mathbf{u})= & \left\{\left[(\boldsymbol{\Lambda}-\mathbf{Q}) \tilde{\boldsymbol{\Psi}}(u)-\mathbf{C} \tilde{\boldsymbol{\Psi}}^{\prime}(u)+\mathbf{Q}\right][\tilde{\mathbf{\Psi}}(0)]^{-1}\right. \\
& \left.-\int_{0}^{u}\left[(\boldsymbol{\Lambda}-\mathbf{Q}) \tilde{\boldsymbol{\Psi}}(u-t)-\mathbf{C} \tilde{\boldsymbol{\Psi}}^{\prime}(u-t)+\mathbf{Q}\right][\tilde{\mathbf{\Psi}}(0)]^{-1} \boldsymbol{\Delta} e^{-\boldsymbol{\Delta} t} d t\right\} \\
& \times\left\{\tilde{\boldsymbol{\Psi}}(u)[\tilde{\mathbf{\Psi}}(0)]^{-1}-\int_{0}^{u} \tilde{\boldsymbol{\Psi}}(x)[\tilde{\boldsymbol{\Psi}}(0)]^{-1} \boldsymbol{\Delta} e^{-\boldsymbol{\Delta}(u-x)} d x\right\}^{-1} .
\end{aligned}
$$

Obviously, $\boldsymbol{\Phi}(u ; \gamma)$ satisfies the boundary condition $\boldsymbol{\Phi}(\infty ; \gamma)=\mathbf{1}$, where $\mathbf{1}=$ $(1,1, \cdots, 1)^{T}$ is a $d \times 1$ column vector.

Remark 2.1. When $d=1$ the model reduces to the model considered in Albrecher and Hipp (2007); $\mathbf{Q}=0$, and then $\mathbf{A}(u)=\boldsymbol{\Lambda}-\mathbf{C} \tilde{\Psi}^{\prime}(u)[\tilde{\Psi}(u)]^{-1}$. In this case, (2.11) simplifies to

$$
\mathbf{C}(\gamma) \boldsymbol{\Phi}^{\prime}(u ; \gamma)=\mathbf{C} \tilde{\boldsymbol{\Psi}}^{\prime}(u)[\tilde{\boldsymbol{\Psi}}(u)]^{-1} \boldsymbol{\Phi}(u ; \gamma),
$$

which can be found in Albrecher and Hipp (2007). 


\section{The expected discounted total tax payment}

For $0 \leq u \leq l$ and $i, j \in \mathbb{J}$, define

$$
L_{i j}(u, l)=\mathrm{E}\left[e^{-\delta \tau_{l}} \chi\left(\tau_{l}<\tau, J\left(\tau_{l}\right)=j\right) \mid R(0)=u, J(0)=i\right],
$$

where $\delta>0$ is the discount factor. $L_{i j}(u, l)$ can be interpreted as the expected present value of one dollar payable at time of reaching the level $l$ in state $j$ without ruin, given that the initial state is $i$ and initial surplus is $u$. Alternatively, it can be viewed as the Laplace transform of the time to reach the level $l$ without ruin, with respect to the parameter $\delta$. Let $\mathbf{L}(u, l)=\left(L_{i j}(u, l)\right)_{d \times d}$ be a matrix, from Li and $\mathrm{Lu}$ (2008) we have $\mathbf{L}(l, l)=\mathbf{I}$ and

$$
\mathbf{L}(u, l)=\mathbf{v}_{\delta}(u)\left[\mathbf{v}_{\delta}(l)\right]^{-1},
$$

where $\mathbf{v}_{\delta}(u)=\left(v_{i j}(u ; \delta)\right)_{d \times d}$ is an matrix whose columns satisfy the system of integro-differential equations

$$
c_{i} v_{i}^{\prime}(u ; \delta)=\left(\lambda_{i}+\delta\right) v_{i}(u ; \delta)-\lambda_{i} \int_{0}^{u} v_{i}(u-x ; \delta) d F_{i}(x)-\sum_{k=1}^{d} q_{i k} v_{k}(u ; \delta),
$$

with boundary conditions $v_{i j}(0 ; \delta)=\chi(i=j)$ for $i, j \in \mathbb{J}$.

Now we modify the surplus process (1.1) by the payment of dividends according to a constant barrier strategy: when the surplus exceeds a constant barrier $l(\geq u)$, dividends are paid continuously so the surplus stays at level $l$ until a new claim occurs. Under such a modified model, let $V_{i j}(u, l)$ be the expected present value of dividend payment before ruin if ruin is caused by a claim in state $j$ given the initial state $i$ and initial surplus $u$. Let $\mathbf{V}(u, l)=\left(V_{i j}(u, l)\right)_{d \times d}$ be a matrix. It follows from $\mathrm{Li}$ and $\mathrm{Lu}(2008)$ that

$$
\mathbf{V}(u, l)=\mathbf{v}_{\delta}(u)\left[\mathbf{v}_{\delta}^{\prime}(l)\right]^{-1}
$$

From (3.1) and (3.2), we have

$$
\mathbf{L}(u, l)=\mathbf{V}(u, l)[\mathbf{V}(l, l)]^{-1} .
$$

Let $D_{i}(u)$ be the expected accumulated discounted tax until ruin for given initial state $i \in \mathbb{J}$ and initial surplus $u \geq 0$. Similar to Albrecher and Hipp (2007), we want to express the results for $\mathbf{D}(u):=\left(D_{1}(u), D_{2}(u), \cdots, D_{d}(u)\right)^{T}$ in terms of $\mathbf{V}$. Next we will show a differential equation satisfied by $\mathbf{D}(u)$. 
Theorem 3.1. For $u \geq 0, \mathbf{D}(u)$ satisfies the following differential equation:

$$
\mathbf{C}(\gamma) \mathbf{D}^{\prime}(u)=\mathbf{C}[\mathbf{V}(u, u)]^{-1} \mathbf{D}(u)-\mathbf{C} \boldsymbol{\gamma},
$$

with boundary condition

$$
\mathbf{D}(\infty)=\mathbf{V}(\infty, \infty) \boldsymbol{\gamma}
$$

Proof. First, for each $i \in \mathbb{J}$, we derive the integro-differential equation satisfied by $D_{i}(u)$. Similar to the proof of Theorem 2.1, we have

$$
\begin{aligned}
D_{i}(u)= & \int_{0}^{\infty} q_{i} e^{-q_{i} t} d t \int_{0}^{t} \lambda_{i} e^{-\lambda_{i} s} d s\left[\int_{0}^{s} c_{i} \gamma_{i} e^{-\delta x} d x+e^{-\delta s}\right. \\
& \left.\times \int_{0}^{u+c_{i}\left(1-\gamma_{i}\right) s} L_{i j}\left(u+c_{i}\left(1-\gamma_{i}\right) s-x, u+c_{i}\left(1-\gamma_{i}\right) s\right) D_{j}\left(u+c_{i}\left(1-\gamma_{i}\right) s\right) d F_{i}(x)\right] \\
& +\int_{0}^{\infty} \lambda_{i} e^{-\lambda_{i} s} \int_{0}^{s} q_{i} e^{-q_{i} t}\left[\int_{0}^{t} c_{i} \gamma_{i} e^{-\delta x} d x+e^{-\delta t} \sum_{k \neq i} \frac{q_{i k}}{q_{i}} D_{k}\left(u+c_{i}\left(1-\gamma_{i}\right) t\right)\right] d t d s \\
= & \int_{0}^{\infty} \lambda_{i} e^{-\left(\lambda_{i}+q_{i}+\delta\right) s} \int_{0}^{u+c_{i}\left(1-\gamma_{i}\right) s} L_{i j}\left(u+c_{i}\left(1-\gamma_{i}\right) s-x, u+c_{i}\left(1-\gamma_{i}\right) s\right) \\
& \times D_{j}\left(u+c_{i}\left(1-\gamma_{i}\right) s\right) d F_{i}(x) d s+\int_{0}^{\infty} e^{-\left(\lambda_{i}+q_{i}+\delta\right) t} \sum_{k \neq i} q_{i k} D_{k}\left(u+c_{i}\left(1-\gamma_{i}\right) t\right) d t \\
& +\frac{c_{i} \gamma_{i}}{\lambda_{i}+q_{i}+\delta} .
\end{aligned}
$$

Changing variables $w=u+c_{i}\left(1-\gamma_{i}\right) t$ (or $w=u+c_{i}\left(1-\gamma_{i}\right) s$ ) leads to

$$
\begin{aligned}
D_{i}(u)= & \int_{u}^{\infty} \frac{\lambda_{i}}{c_{i}\left(1-\gamma_{i}\right)} e^{-\left(\lambda_{i}+q_{i}+\delta\right) \frac{w-u}{c_{i}\left(1-\gamma_{i}\right)}} \int_{0}^{w} L_{i j}(w-x, w) D_{j}(w) d F_{i}(x) d w \\
& +\int_{u}^{\infty} \frac{1}{c_{i}\left(1-\gamma_{i}\right)} e^{-\left(\lambda_{i}+q_{i}+\delta\right) \frac{w-u}{c_{i}\left(1-\gamma_{i}\right)}} \sum_{k \neq i} q_{i k} D_{k}(w) d w+\frac{c_{i} \gamma_{i}}{\lambda_{i}+q_{i}+\delta}(3.6)
\end{aligned}
$$

Differentiating the above equation with respect to $u$ gives

$$
\begin{aligned}
c_{i}\left(1-\gamma_{i}\right) D_{i}^{\prime}(u)= & \left(\lambda_{i}+\delta\right) D_{i}(u)-\lambda_{i} \int_{0}^{u} L_{i j}(u-x, u) D_{j}(u) d F_{i}(x) \\
& -\sum_{k=1}^{d} q_{i k} D_{k}(u)-c_{i} \gamma_{i} .
\end{aligned}
$$

In matrix form, we have

$$
\mathbf{C}(\gamma) \mathbf{D}^{\prime}(u)=(\boldsymbol{\Lambda}+\delta \mathbf{I}-\mathbf{Q}) \mathbf{D}(u)-\int_{0}^{u} \tilde{\mathbf{\Lambda}}(x) \mathbf{L}(u-x, u) d x D(u)-\mathbf{C} \boldsymbol{\gamma} .
$$

Then from (3.3) we have

$$
\mathbf{C}(\gamma) \mathbf{D}^{\prime}(u)=(\boldsymbol{\Lambda}+\delta \mathbf{I}-\mathbf{Q}) \mathbf{D}(u)-\int_{0}^{u} \tilde{\boldsymbol{\Lambda}}(x) \mathbf{V}(u-x, u) d x[\mathbf{V}(u, u)]^{-1} \mathbf{D}(u)-\mathbf{C} \boldsymbol{\gamma}
$$


From Li and Lu (2007) and (2008), we have

$$
\int_{0}^{u} \tilde{\Lambda}(x) \mathbf{V}(u-x, u) d x=(\mathbf{\Lambda}+\delta \mathbf{I}-\mathbf{Q}) \mathbf{V}(u, u)-\mathbf{C} .
$$

Then (3.4) follows from the equations (3.7) and (3.8).

Using the same techniques of Albrecher and Hipp (2007), we know $D_{i}(u)$ is bounded for all $u \geq 0$ and $i \in \mathbb{J}$. Taking limit $u \rightarrow \infty$ in (3.6) yields (using de'l Hopital's rule),

$$
\left(\lambda_{i}+\delta\right) D_{i}(\infty)-\sum_{k=1}^{d} q_{i k} D_{k}(\infty)-\lambda_{i} \int_{0}^{\infty} L_{i j}(\infty-x, \infty) f_{i}(x) d x D_{j}(\infty)=c_{i} \gamma_{i},
$$

or in the matrix notation

$$
(\boldsymbol{\Lambda}+\delta \mathbf{I}-\mathbf{Q}) \mathbf{D}(\infty)-\int_{0}^{\infty} \tilde{\mathbf{\Lambda}}(x) \mathbf{L}(\infty-x, \infty) d x \mathbf{D}(\infty)=\mathbf{C} \boldsymbol{\gamma}
$$

Then the boundary condition (3.5) follows from equations (3.3), (3.8) and (3.9).

\section{Analytical expressions for $\boldsymbol{\Phi}(u ; \gamma)$ and $\mathbf{D}(u)$}

For $u>0$, we derive the analytical expressions for non-ruin probabilities $\boldsymbol{\Phi}(u ; \gamma)$ and the expected accumulated discounted tax until ruin $\mathbf{D}(u)$ by iteration.

From (2.5) we can write

$$
\mathbf{C v}^{\prime}(u)=(\boldsymbol{\Lambda}-\mathbf{Q}) \mathbf{v}(u)-\int_{0}^{u} \tilde{\Lambda}(x) \mathbf{v}(u-x) d x .
$$

Together with (2.4) and (2.7), we have

$$
\boldsymbol{\Gamma} \boldsymbol{\Phi}^{\prime}(u ; \gamma)=\mathbf{v}^{\prime}(u)[\mathbf{v}(u)]^{-1} \boldsymbol{\Phi}(u ; \gamma)
$$

where $\boldsymbol{\Gamma}=\operatorname{diag}\left(1-\gamma_{1}, \cdots, 1-\gamma_{d}\right)$. Let $\hat{\mathbf{\Phi}}(u ; \gamma):=\boldsymbol{\Phi}\left(\frac{1}{u} ; \gamma\right)$ and $\hat{\mathbf{v}}(u):=\mathbf{v}\left(\frac{1}{u}\right)$, then (4.2) yields

$$
\Gamma \hat{\boldsymbol{\Phi}}^{\prime}(u ; \gamma)=\hat{\mathbf{v}}^{\prime}(u)[\hat{\mathbf{v}}(u)]^{-1} \hat{\boldsymbol{\Phi}}(u ; \gamma)
$$

and $\hat{\boldsymbol{\Phi}}(0 ; \gamma)=\boldsymbol{\Phi}(\infty ; \gamma)=\mathbf{1}$. We replace $u$ by $x$ in $(4.3)$ and then integrate both sides of the equation from 0 to $u$ with respect to $x$. Thus, we obtain

$$
\hat{\mathbf{\Phi}}(u ; \gamma)=\mathbf{1}+\int_{0}^{u} \boldsymbol{\Gamma}^{-1} \hat{\mathbf{v}}^{\prime}(x)[\hat{\mathbf{v}}(x)]^{-1} \hat{\boldsymbol{\Phi}}(x ; \gamma) d x .
$$


Let

$$
\hat{\mathbf{\Phi}}_{n}(u ; \gamma)=\mathbf{1}+\int_{0}^{u} \boldsymbol{\Gamma}^{-1} \hat{\mathbf{v}}^{\prime}(x)[\hat{\mathbf{v}}(x)]^{-1} \hat{\mathbf{\Phi}}_{n-1}(x ; \gamma) d x, \quad n=1,2, \cdots,
$$

with

$$
\hat{\boldsymbol{\Phi}}_{0}(u ; \gamma)=\mathbf{1}
$$

and

$$
\hat{\boldsymbol{\phi}}_{n}(u ; \gamma)=\hat{\boldsymbol{\Phi}}_{n}(u ; \gamma)-\hat{\boldsymbol{\Phi}}_{n-1}(u ; \gamma), \quad n=1,2, \cdots
$$

with

$$
\hat{\phi}_{0}(u ; \gamma)=1
$$

Assume that $\hat{\mathbf{v}}^{\prime}(x)[\hat{\mathbf{v}}(x)]^{-1}$ is continuous in $0 \leq x \leq u \leq K<\infty$, where $K$ is a constant that can be chosen arbitrary large. By Theorem 3.11 of Linz (1985), we know that the unique solution of $\hat{\boldsymbol{\Phi}}(u ; \gamma)$ is given by

$$
\hat{\boldsymbol{\Phi}}(u ; \gamma)=\sum_{n=0}^{\infty} \hat{\boldsymbol{\phi}}_{n}(u ; \gamma)
$$

Thus for $u>0$, we have

$$
\boldsymbol{\Phi}(u ; \gamma)=\hat{\boldsymbol{\Phi}}\left(\frac{1}{u} ; \gamma\right)=\sum_{n=0}^{\infty} \hat{\boldsymbol{\phi}}_{n}\left(\frac{1}{u} ; \gamma\right)
$$

From (3.2) and (3.4) we have

$$
\Gamma \mathbf{D}^{\prime}(u)=\mathbf{v}_{\delta}^{\prime}(u)\left[\mathbf{v}_{\delta}(u)\right]^{-1} \mathbf{D}(u)-\gamma .
$$

Let $\mathbf{Z}(u)=\left(z_{i j}(u)\right)_{d \times d}$ is an matrix whose columns $\mathbf{Z}_{j}=\left(z_{1 j}, \cdots, z_{d j}\right)^{T}, j=$ $1, \cdots, d$, are particular solutions to the following system of integro-differential equations:

$$
\Gamma \mathbf{Z}_{j}^{\prime}(u)=\mathbf{v}_{\delta}^{\prime}(u)\left[\mathbf{v}_{\delta}(u)\right]^{-1} \mathbf{Z}_{j}(u)
$$

with boundary condition $\mathbf{Z}(\infty)=\mathbf{I}$. It easy to verify that

$$
\mathbf{D}(u)=\mathbf{Z}(u) \mathbf{D}(\infty)+\int_{u}^{\infty} \mathbf{Z}\left(\frac{1}{x-u}\right) d x \boldsymbol{\gamma}
$$

Let $\hat{\mathbf{Z}}(u):=\mathbf{Z}\left(\frac{1}{u}\right)$ and $\hat{\mathbf{v}}_{\delta}(u):=\mathbf{v}_{\delta}\left(\frac{1}{u}\right)$, then $\hat{\mathbf{Z}}(0)=\mathbf{I}$. Let

$$
\hat{\mathbf{Z}}_{n}(u)=\mathbf{I}+\int_{0}^{u} \boldsymbol{\Gamma}^{-1} \hat{\mathbf{v}}_{\delta}^{\prime}(x)\left[\hat{\mathbf{v}}_{\delta}(x)\right]^{-1} \hat{\mathbf{Z}}_{n-1}(x) d x, \quad n=1,2, \cdots,
$$

with

$$
\hat{\mathbf{Z}}_{0}(u)=\mathbf{I},
$$


and

$$
\hat{\boldsymbol{\psi}}_{n}(u)=\hat{\mathbf{Z}}_{n}(u)-\hat{\mathbf{Z}}_{n-1}(u), \quad n=1,2, \cdots
$$

with

$$
\hat{\boldsymbol{\psi}}_{0}(u)=\mathbf{I}
$$

Similarly, for $u>0, \mathbf{Z}(\mathrm{u})$ can be written as

$$
\mathbf{Z}(u)=\hat{\mathbf{Z}}\left(\frac{1}{u}\right)=\sum_{n=0}^{\infty} \hat{\boldsymbol{\psi}}_{n}\left(\frac{1}{u}\right) .
$$

Note that $\mathbf{v}(u)=\mathbf{v}_{0}(u)$ and form $\mathrm{Li}$ and $\mathrm{Lu}(2007)$ and $\mathrm{Lu}$ and $\mathrm{Li}$ (2008) we know that

$$
\mathbf{v}_{\delta}(z)=\mathcal{L}^{-1}\left\{\left[s \mathbf{I}-\mathbf{C}^{-1}(\boldsymbol{\Lambda}+\delta \mathbf{I}-\mathbf{Q})+\mathbf{C}^{-1} \hat{\boldsymbol{\Lambda}}(s)\right]^{-1}\right\}, \quad z \geq 0
$$

where $\hat{\boldsymbol{\Lambda}}(s)=\operatorname{diag}\left(\lambda_{1} \hat{f}_{1}(y), \lambda_{2} \hat{f}_{2}(y), \cdots, \lambda_{n} \hat{f}_{n}(y)\right)$ is the Laplace transform of $\tilde{\boldsymbol{\Lambda}}$ and $\mathcal{L}^{-1}\{\cdot\}$ is the Laplace inversion.

\section{Acknowledgement}

H. Yang would like to acknowledge the Research Grants Council of the Hong Kong Special Administrative Region, China (project No. HKU 7540/08H).R. Wang would like to acknowledge the National Natural Science Foundation of China (10671072), the Doctoral Program Foundation of the Ministry of Education of China (20060269016), and the National Basic Research Program of China (973 Program) under grant number 2007CB814904.

\section{References}

Albrecher, H., Hipp, C., 2007. Lundberg's risk process with tax. Blätter der DGVFM 28(1), 13-28.

Asmussen, S., 1989. Risk theory in a Markovian environment. Scandinavian Actuarial Journal, 69-100.

Cramér, H., 1930. On the Mathematical Theory of Risk. Skandia Jubilee Volume, Stockholm. 
Gerber, H. and Shiu E.S.W., 1998. On the time value of ruin. North American Actuarial Journa 12(1), 48-72; Discussions, 72-78.

Linz, P., 1985. Analystical and Numerical Methods for Volterra Equations. Studies 7, SIAM Studies in Aplied Mathematocs: Philadelpha.

Li, S., Lu, Y., 2007. Moments of the dividend payments and related problems in a Markov-modulated risk model. North American Actuarial Journal 11 (2), 65-76.

Li, S., Lu, Y., 2008. The decompositions of the discounted penalty functions and dividends-penalty identity in a Markov-modulated risk model. ASTIN Bulletin 38(1), 53-71.

Lu, Y., Li, S., 2005. On the probability of ruin in a Markov-modulated risk model. Insurance: Mathematics and Economics 37, 522-532.

Lu, Y., Li, S., 2008. The Markovian regime-switching risk model with a threshold dividend strategy. Insurance: Mathematics and Economics (Inpress).

Lundberg, F. 1909. Uber die theorie der rückversicherung. Transactions of VI International Congress of Actuaries 1, 877-948.

Ng, A., Yang, H., 2006. On the joint distribution of surplus prior and immediately after ruin under a Markovian regime switching model. Stochastic Processes and their Applications 116, 244-266.

Reinhard, J.M., 1984. On a class of semi-Markov risk models obtained as classical risk models in a Markovian enviroment. Astin Bulletin 14, 23-43.

Zhu, J., Yang, H., 2008. Ruin theory for a Markov regime-switching model under a threshold dividend strategy. Insurance: Mathematics and Economics 42, 311318. 